\title{
Herding catalysts at UCSF
}

\section{By Lev Osherovich, Senior Writer}

AstraZeneca plc has a burgeoning network of public-private partnerships, but until now the pharma has lacked a presence in the San Francisco Bay Area, one of the U.S.'s major hubs of life sciences innovation. AstraZeneca now has remedied that, as its MedImmune LLC biologics unit is piggybacking onto an NIH-sponsored initiative to support translational research at the University of California, San Francisco.

MedImmune will become the first industry sponsor of UCSF's Catalyst Awards, a grant program for researchers interested in translational applications of their work. The Catalyst Awards first launched in 2010 and previously were funded entirely by the NIH's Clinical and Translational Science Awards (CTSA) program.

MedImmune will work with UCSF's CTSA administrator, the Clinical and Translational Science Institute (CTSI), to identify and fund translational research and build a portfolio of collaborations at UCSF.

"This is a three-year collaboration with the CTSI at UCSF," said Bing Yao, SVP of respiratory, inflammation and autoimmunity

"Our goal has been to leverage the variety of expertise in the Bay Area. Our consultants and advisors create a virtual incubator for UCSF researchers interested in commercialization of their basic research."

- June Lee,

University of California, San Francisco R\&D at MedImmune. "This collaboration is different than our other collaborations in that it is focused exclusively on translational science. This also gives us access to research in the Bay Area."

June Lee, a professor of medicine at UCSF, said that the Catalyst program aims to provide UCSF researchers with funds to explore the commercial or translational applications of their basic research. Lee is director of Early Translational Research at CTSI.

"We have two calls for proposals annually and four tracks for each cycle-therapeutics, diagnostics, devices and digital health," said Lee. MedImmune will sponsor the therapeutics track.

Financial terms of the partnership were not disclosed, but Yao and Lee said that the company will have the option to in-license or independently finance Catalyst Award projects.
Yao said that MedImmune will have representatives on the Catalyst Award selection committee and will provide access to company resources and expertise to grant recipients.

\section{Translational turf}

Over the last three years, AstraZeneca has built an extensive network of academic collaborations geared toward filling the pharma's early pipeline. In 2013, AstraZeneca led the industry in forming public-private partnerships (PPPs), entering at least 14 disclosed PPPs with a variety of academic institutions worldwide. ${ }^{1}$

Among MedImmune's academic partnerships were a 5-year, \$6.5 million collaboration with The Johns Hopkins University and a $\$ 7$ million deal with the University of Maryland, Baltimore. Both of those partnerships focus on discovery and evaluation of new biologics across a broad range of disease areas.

Although MedImmune will be the first company to provide money to the Catalyst Awards, CTSI itself does have other ties to industry.

In January, CTSI partnered with Quest Diagnostics Inc. to research, develop and validate laboratory-created tests for oncology, neurology and women's health.

Quest will provide up to $\$ 0.5$ million each for 2 projects by UCSF researchers. One project involves using Quest's microarray-based genetic profiling platform to discover and validate biomarkers for autism spectrum disorder (ASD). The other project focuses on identifying biomarkers of favorable drug response in brain cancer.

UCSF is eligible for royalties and services developed by Quest based on the joint projects. Lee said that the details of a third project partnered with Quest will be disclosed shortly.

Lee said that in addition to handing out Catalyst Award money, CTSI has built a network of about 140 biopharma consultants in the San Francisco Bay Area. These consultants work with Catalyst Award recipients and other UCSF researchers to plan discovery and pre-IND studies for translational projects.

"Our goal has been to leverage the variety of expertise in the Bay Area," said Lee. "Our consultants and advisors create a virtual incubator for UCSF researchers interested in commercialization of their basic research."

Osherovich, L. SciBX 7(8); doi:10.1038/scibx.2014.216

Published online Feb. 27, 2014

\section{REFERENCES}

1. Edelson, S. \& Lou, K.-J. SciBX 7(7); doi:10.1038/scibx.2014.188

\section{COMPANIES AND INSTITUTIONS MENTIONED}

AstraZeneca plc (LSE:AZN; NYSE:AZN), London, U.K.

The Johns Hopkins University, Baltimore, Md.

MedImmune LLC, Gaithersburg, Md.

National Institutes of Health, Bethesda, Md.

Quest Diagnostics Inc. (NYSE:DGX), Madison, N.J.

University of California, San Francisco, Calif.

University of Maryland, Baltimore, Md. 